EPJ Web of Conferences 19, 02008 (2012)

DOI: $10.1051 /$ epjconf/20121902008

(C) Owned by the authors, published by EDP Sciences, 2012

\title{
High-velocity streams in the Milky Way halo with the SDSS and GSC-II kinematic survey
}

\author{
P. Re Fiorentin ${ }^{1, a}$, M.G. Lattanzi ${ }^{1}$, R.L. Smart ${ }^{1}$, A. Spagna1 ${ }^{1}$, T.C. Beers ${ }^{2,3}$ \\ and A. Curir ${ }^{1}$ \\ ${ }^{1}$ INAF - Astronomical Observatory of Torino, Italy \\ ${ }^{2}$ National Optical Astronomy Observatory, Tucson, AZ, USA \\ ${ }^{3}$ Michigan State University and JINA, E. Lansing, MI, USA
}

\begin{abstract}
We explore the stellar halo of the Milky Way to search for fossil signatures of past mergers, consisting of streams of particles torn from their progenitors during the process of hierarchical merging. We examine a representative sample of about 2700 halo subdwarfs in the solar neighborhood, selected from a new kinematic survey produced by means of spectrophotometric data from the Sloan Digital Sky Survey, and high-quality proper motions derived from multi-epoch positions using the Guide Star Catalog II. We find statistical evidence for discrete overdensities, likely possible accretion remnants. This methodology and the quality of the selected sample are an efficient means to discover new members of known streams and to identify new streams themselves, and set the basis for future studies.
\end{abstract}

\section{INTRODUCTION}

In a CDM Universe, galaxies like the Milky Way grow by mergers of dwarf galaxies; this theory predicts the presence of substructures due to accretion events experienced over their lifetime. Indeed, recent observations have shown that considerable structure is still present in the halo of the Milky Way, indicating that such events have had role in its formation history (e.g., [1, 2]).

The Galactic halo may retain memory of its merging history in the form of fossil streams of stars which, although sometimes of very low spatial density, may be detectable as stellar groups with coherent kinematics and metallicities. However, recovering fossil structures in the solar neighborhood is considerably more difficult - timescales are very short in this region of the Galaxy, and streams can easily overlap in space. This degeneracy can only be broken with 6-D (phase-space) or 7-D (including abundances) information achievable only by integrating astrometry, photometry, and spectroscopy.

\section{METHODS}

The SDSS and GSC-II Kinematic Survey is produced to serve this task. It provides accurate spectroscopic parameters (effective temperature, surface gravity, metallicity, and radial velocity), proper motions, and distance estimates for about 27000 FGK subdwarfs (e.g., [5]).

Motivated by results from high-resolution CDM simulations and previous analysis ([4]), we started using this sample to explore the stellar halo of the Milky Way, and look for kinematic substructures. This is implemented by selecting and analysing subsamples of stellar tracers of the full 7-D phase-space of the Galactic halo population(s), based on objects that pass within a few kpcs of the Sun.

\footnotetext{
ae-mail: re_fiorentin@oato.inaf .it
}

This is an Open Access article distributed under the terms of the Creative Commons Attribution-Noncommercial License 3.0, which permits unrestricted use, distribution, and reproduction in any noncommercial medium, provided the original work is properly cited. 

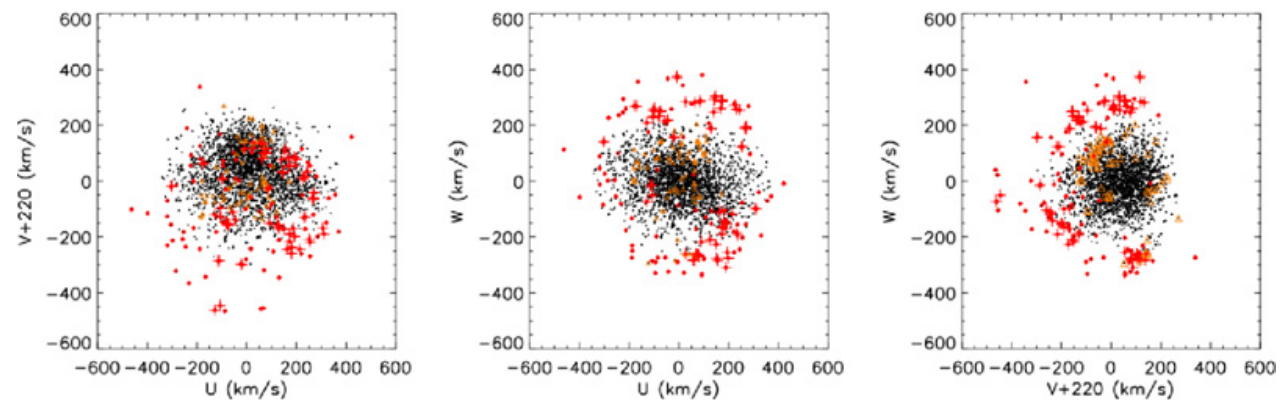

Figure 1. Velocity projections in UVW space. Of the 2709 subdwarfs, the $5 \%$ fastest moving are highlighted (red circles). Among them, the crosses identify groups with velocity difference less than $42 \mathrm{kms}^{-1}$. Triangles show known streams as listed in [3].

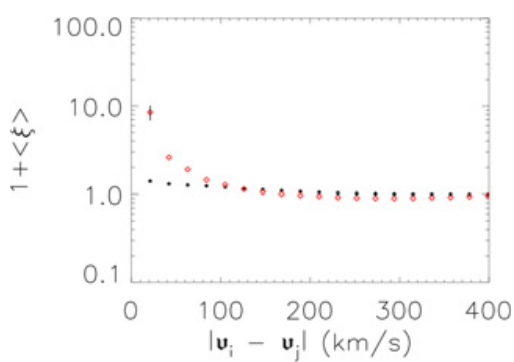

Figure 2. Correlation function for the full sample (dots), and its $5 \%$ fastest-moving subset (diamonds).

While the velocity distribution of the subset of 2709 halo stars with $[\mathrm{Fe} / \mathrm{H}]<-1.5$ within $3 \mathrm{kpc}$ is relatively smooth, the kinematics of the $5 \%$ fastest moving stars appear more clumped and anisotropic, as shown in Fig. 1. Deviations from a smooth Gaussian distribution due to kinematic substructures are quantified by computing the two-point velocity correlation function, $\xi$, which measures the excess of pairs of stars moving with a given velocity difference, above that expected from a representative random sample.

Clumping due to kinematic substructures (i.e., groups of stars moving with similar velocities) appears as an excess at small velocity difference (see Fig. 2). This signal, clearly much stronger for the subset of $5 \%$ fastest objects, is due to moving groups formed by stars denoted by the crosses in Fig. 1.

\section{CONCLUSIONS}

We have found statistical evidence of substructure in the space motions of the fastest moving stars. This appears to be due to a small number of moving groups that are strongly clustered also in the angularmomentum phase space. This methodology is able to provide additional subdwarf members and streams to the results of previous analysis (e.g., [3]).

Further investigation of the group members by their intrinsic properties (e.g., chemical abundance and orbits) suggest that they may be possible fossil remnants from the outer halo currently in the solar neighborhood. 
Assembling the Puzzle of the Milky Way

\section{References}

[1] Helmi, A. et al., Nature, 402, (1999) 53

[2] Ibata, R. et al., Nature, 370, (1994), 194

[3] Klement, R. et al., ApJ, 698, (2009) 865

[4] Re Fiorentin, P., et al., A\&A, 439, (2005) 551

[5] Spagna, A. et al., A\&A, 510, (2010) L4 